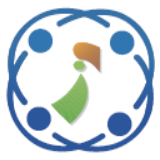

\title{
DTCWT-ICA based Improved DOA Estimation of Closely Spaced and Coherent Signals with Uniform Linear Array
}

\author{
Dharmendra Ganage ${ }^{1 *} \quad$ Yerram Ravinder $^{2}$ \\ ${ }^{1}$ Department of Electronics and Telecommunication, Sinhgad College of Engineering, Pune, India \\ ${ }^{2}$ Department of Electronics and Telecommunication, Pune Institute of Computer Technology, Pune, India \\ *Corresponding author's Email: dgganage.scoe@sinhgad.edu
}

\begin{abstract}
Direction of Arrival (DOA) estimation is the important stage in a smart antenna that estimates the direction of impinging signals on an antenna array, and the most high-resolution DOA estimation subspace algorithm is Multiple Signal Classification (MUSIC). MUSIC algorithm is best suited for estimating the non-coherent signals due to its accuracy and a high degree of resolution. However, its performance degrades in presence of multipath propagation that has coherent and closely spaced signals and at low Signal to Noise Ratio. Many researchers proposed modifications to the MUSIC algorithm to improve its performance even under multipath, coherent and closely spaced signal environment. This paper focuses on investigating a proposed preprocessing technique, referred to as CW-ICA MUSIC that improves the performance of the MUSIC algorithm under above scenario even at low SNR. Simulation results confirm that the proposed preprocessing technique based on Dual Tree Complex Wavelet Transform (DTCWT) and Independent Component Analysis (ICA) outperforms the reported modified MUSIC algorithms such as Wavelet-MUSIC, and SS-MUSIC.
\end{abstract}

Keywords: DOA, MUSIC, DTCWT, ICA, Closely spaced, Coherent signal.

\section{Introduction}

Explosive growth of subscribers to wireless communication systems demands increased capacity, data rate and bandwidth. As a result, many technologies have been proposed to improve the capacity of wireless communication systems. One of the promising technologies that have been proposed in recent times is Smart Antenna. Smart antenna technology is an adaptive array system that utilizes various digital signal processing algorithms to detect the location of the user by estimation direction of arrival of the signal and beamforming accordingly [1]. The DOA estimation algorithms play a vital role in smart antenna in estimating the direction of arrival of impinging signals on antenna array, and supporting the beamforming algorithms to synthesize the radiation pattern to detect the desired signals and nullify the undesired signals.

Many DOA estimation techniques have been proposed in literature. Multiple Signal Classification
(MUSIC) and Estimation of Signal Parameter via Rotational Invariance Techniques (ESPRIT) are the most widely adopted classical high resolution subspace based algorithms [2, 3]. The basic concepts used in subspace algorithms are eigenanalysis of the correlation matrix. The effectiveness of the subspace algorithm is lost when the received signals are closely spaced and coherent. These algorithms work with high SNR, more antenna sensors and a large number of snapshots. In case of multipath propagation, the signal becomes coherent and closely spaced, results in deterioration of the performance of subspace-based DOA estimation algorithms [4].

DOA estimation of multipath reception of uncorrelated and coherent signals have been presented in [5], that groups the coherent signals by generating the cross term effects between coherent groups by exploiting high probability failure of DOA estimation for coherent signals. DOA estimation has been carried out in the multipath 
reception of the uncorrelated and coherent signals explored by [6] removes cross term effect. These techniques do not perform well for detection of the uncorrelated signal for low SNR and less number of snapshots.

The wavelet operator is utilized to enhance the performance of MUSIC, and ESPRIT algorithms [7]. However, it correctly detects the closely spaced signals only under relatively higher and positive SNR conditions. Multipath DOA estimation method presented in [8] utilizes the combination of complex fast independent component analysis and compressed sensing. A resolution of $2^{\circ}$ is achieved for direct and multipath components with relatively high SNR of $20 \mathrm{~dB}$.

A complex-value based fast ICA algorithm that extracts the features of Gaussian noise from nonGaussian signal components is presented in [9]. The performance for correlated signal with resolution of $2^{\circ}$ is achieved with SNR of $16 \mathrm{~dB}$. DOA estimation for uncorrelated and coherent signals presented in [10] utilizes ICA and several parameter estimations. The results were demonstrated for coherent signals with SNR of 10dB.

The application of blind source separation using ICA based DOA estimation has been studied in [11]. The independent component analysis and forwardbackward spatial smoothing techniques is used for DOA estimation [12]. With SNR of 3dB and 1000 snapshots were utilized for $5^{\circ}$ resolution of coherent signal estimation. An improved method is proposed in [13] to distinguish between uncorrelated and coherent signals. The results are demonstrated for SNR of $-4 \mathrm{~dB}$.

DOA estimation of two closely spaced uncorrelated sources proposed by combining spatial sampling and MUSIC algorithm referred to as SSMUSIC is reported in [14]. The proposed method improves the resolution but the accuracy needs to be further improved compared to the reported accuracy for the angular separation less than $4^{0}$.

DOA estimation algorithms need to be modified when the signals are closely spaced and coherent with $2^{\circ}$ resolution with relatively very low SNR for the performance enhancement. Most of the DOA estimation methods designed for non-coherent signals or coherent but under high SNR conditions [5-14]. The signals are closely spaced due to multipath propagation; they usually become coherent. The main reason for the degradation of performance is because of loss of coherence matrix rank.

In this paper, a novel algorithm referred to as CW-ICA MUSIC that utilizes DTCWT and ICA at pre-processing (denoising) stage to improve the performance of the MUSIC algorithm for the closely spaced coherent signals reception. This proposed denoising technique improves the received SNR. ICA is utilized as thresholding technique for removing more significant noisy coefficients after forwarding process of DTCWT. The proposed method performs better even for moderate number of snapshots with few antenna elements, and low SNR.

The organization of paper: Section 2 introduces the array signal model of a uniform linear array. Section 3 describes the proposed method "CW-ICA MUSIC" based on DTCWT and ICA. Section 4 presents the experimental simulation verification of the proposed algorithm, and comparison with basic MUSIC, wavelet-based MUSIC, and SS-MUSIC algorithms. Finally, Section 5 concludes the article.

\section{Array signal model}

The antenna array is an array composed of several antenna elements aligned in a straight line with uniform amplitude and spacing on a plane is referred to as Uniform Linear Array (ULA) shown in Fig. 1.

Let ULA composed of $M$ isotropic antenna array elements with inter-element spacing $d$, and $K$ farfield received narrowband signals from different directions $\theta_{1}, \ldots, \theta_{K}$ with carrier wavelength $\lambda$. It has to be $M>K$, and $d$ is not more than $\lambda / 2$ of the highest frequency. The received noise component of $M$ antenna elements is uncorrelated and assumed to be additive white Gaussian noise with zero mean of variance $\sigma^{2}$.

The array output is calculated by adding the individual antenna array elements. The received signal at $M$ array elements is given by [1],

$$
x_{m}(t)=\sum_{k=1}^{D} a_{m}\left(\theta_{k}\right) S_{k}(t)+n_{m}(t)
$$

Where $S_{k}(t)$ represents the input signal amplitude received at $k^{t h}$ signal and $n_{m}(t)$ is the additive Gaussian noise.

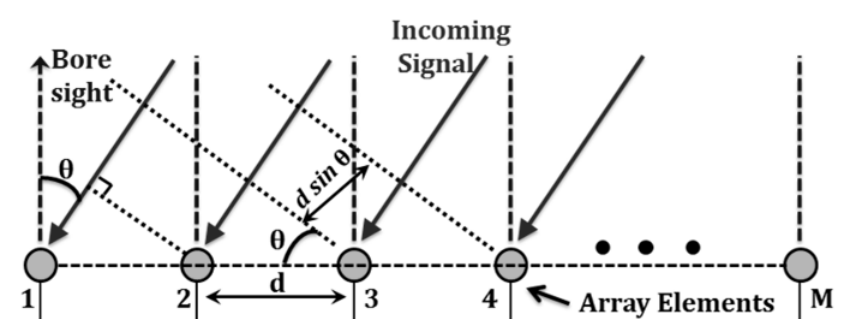

Figure. 1 Antenna array signal model with ' $M$ ' elements 
Received signal in vector form is given by,

$$
X(n)=A S(n)+N(n)
$$

Where,

$$
\begin{aligned}
& X(t)=\left[x_{1}(t), x_{2}(t) \cdots x_{m}(t)\right]^{T}, \\
& S(t)=\left[s_{1}(t), s_{2}(t) \cdots s_{m}(t)\right]^{T}, \\
& N(t)=\left[n_{1}(t), n_{2}(t) \cdots n_{m}(t)\right]^{T} \text { and }
\end{aligned}
$$

$A$ is the Vandermonde matrix of $M \times K$. The antenna array steering vector is,

$$
\begin{aligned}
& a(\theta)=\left[1, e^{-j \varphi 1}, \ldots, e^{-j(M-1) \varphi K}\right]^{T} \text { and }, \\
& \varphi=\frac{2 \pi d}{\lambda} \sin \theta_{k}
\end{aligned}
$$

Where $S(n)$ is the $K \times 1$ signal vector, $N(n)$ is the $M \times 1$ noise vector, superscripts $T$ represents the transpose of a matrix, and $k=1,2, \ldots, K$. Thus, DOA is estimated by calculating the values of $\theta$ from given signal samples or snapshots $(N)$ of array outputs. Now, the array covariance matrix $R_{x}$ is given by,

$$
R_{x}=E\left[X(t) X^{H}(t)\right]=A R_{S} A^{H}+R_{N}
$$

Where, $R_{S}=E\left[s(t) s^{H}(t)\right]$ is the source correlation matrix, $R_{N}=\sigma^{2} I$ is the noise correlation matrix with noise variance $\sigma^{2}, I$ act as $M \times M$ an identity matrix, and $H$ represents the Hermitian or conjugate transpose of a matrix. To estimate the array covariance matrix at the $k^{\text {th }}$ signal of $N$ number of snapshots is given by,

$$
\hat{R}=\frac{1}{N} \sum_{i=1}^{N} X(i) X^{H}(i)
$$

DOA estimation is carried out with the decomposition of an array covariance matrix as per the theory of matrix eigen decomposition. The source correlation matrix $R_{S}$ must be a non-singular matrix, and $M>K$. The rank of $A R_{S} A^{H}$ is the full rank of $K$ matrix.

As per Eq. (4), $R_{x}{ }^{H}=R_{x}$ is the full rank matrix with $D$ positive and real number eigen values, remaining $M-D$ are zero eigen values. The $M$ positive real eigen values $\left(\lambda_{1}, \lambda_{2}, \ldots, \lambda_{M}\right)$ correspond to $M$ eigen vectors $\left(v_{1}, v_{2}, \ldots, v_{M}\right)$. The $R_{x}$ is Hermite matrix, and its eigen values are orthogonal to each other equal to $\sigma^{2}$ and are the minimum eigen values of the matrix $R_{x}$. This eigen decomposition property used in DOA estimation by using subspace-based methods.

\section{Proposed method for DOA estimation using CW-ICA MUSIC algorithm}

The multipath signal has a signal of interest and several non-signal of interest components, which are coherent to each other and closely spaced. In turn, it will combine with the signal of interest and degrade the rank of signal subspace matrix. Resulting estimated performance will decline and may fail for existing DOA estimation techniques such as MUSIC to find the direction of multipath signal and source signal.

Many methods are proposed by researcher for DOA estimation of multipath signals to pre-process the coherent signal to achieve incoherence [8], such as Forward Spatial Smoothing, Forward and backward spatial smoothing, Modified Spatial Smoothing, Toeplitz Algorithm, and Subspace fitting Algorithm. However, incoherence capacity obtained by the expense of loss array aperture, required larger value of SNR, a number of snapshots and generates large estimation error.

Fig. 2 shows the flowchart of the proposed DOA estimation algorithm referred to as CW-ICA MUSIC based on DTCWT and ICA. The DTCWT and ICA are acting as denoising and thresholding stage respectively. The denoised signal is processed by the MUSIC algorithm to estimate the DOAs of received signals.

Firstly, initialized the $M$ (number of antenna elements), $N$ (number of snapshots), SNR then array steering vector is calculated. The array steering vector is converted into wavelet coefficients using DTCWT analysis (forward) decomposition filter. The decomposition signal consists of detail and approximate signal coefficients. The decomposition process of signal creates significantly correlated complex wavelet coefficients in the neighboring region [18]. All the detail and approximation signal coefficients are thresholded by using ICA. The ICA process described in detailed in Fig. 4 to remove the nearby correlated wavelet coefficients. Now, the denoised forward signal is used to reconstruct the denoised signal $\operatorname{ReX}$ through synthesis (inverse) filter. This denoised signal $\operatorname{ReX}$ processed by using the MUSIC algorithm to estimate the DOA of signals. 


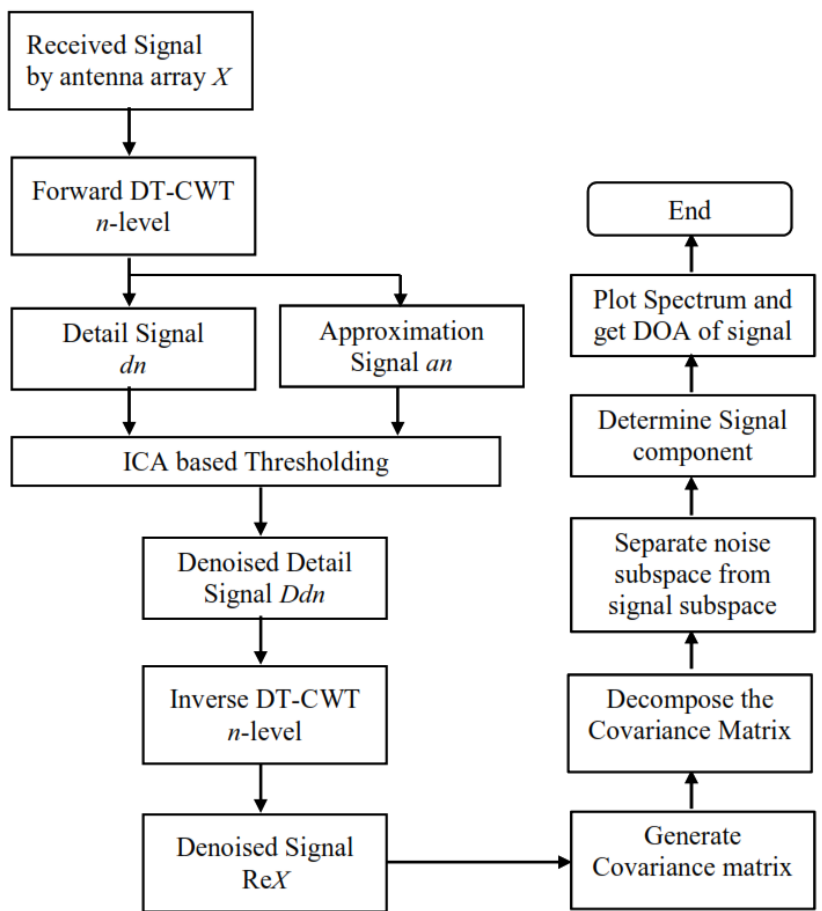

Figure. 2 Flowchart of the proposed method

\subsection{Dual tree complex wavelet transform}

DTCWT is Dual Tree Complex Wavelet Transform is an alternative to Discrete Wavelet Transform (DWT) which does not support shiftinvariance and directional selectivity for $M$ dimensional signals [15]. Shift-invariance property is essential in the process of closely spaced coherent signals detection because of direction information embedded with the large coefficient plane in the form of magnitude and phase relationship.

DTCWT consists of two trees of wavelet filters which produce real part and imaginary part of the complex wavelet. DTCWT can rely on the observation with a real and approximate DWT by enhancing the sampling rate by twice at every level of the tree which can achieve approximate shift invariance. It accomplishes signal decomposition at analysis part, and reconstruction at synthesis part mainly through two parallel real and approximate wavelets ( $h$ tree and $g$ tree). The trees consist of two filters namely: Low Pass Filters $\left(h_{0}\right.$ and $\left.g_{0}\right)$, and High Pass Filters $\left(h_{l}\right.$ and $\left.g_{l}\right)$. The parallel real wavelet filter banks are designed to get a complex signal, i.e., real and imaginary coefficients. These parallel trees form the Hilbert transform pair approximately to each other, and implementation is carried out by using two mother wavelets [15]. Fig. 3 shows the one level DTCWT analysis (decomposition) and synthesis (reconstruction) tree. The odd/even filter bank and Q-shift (quarter

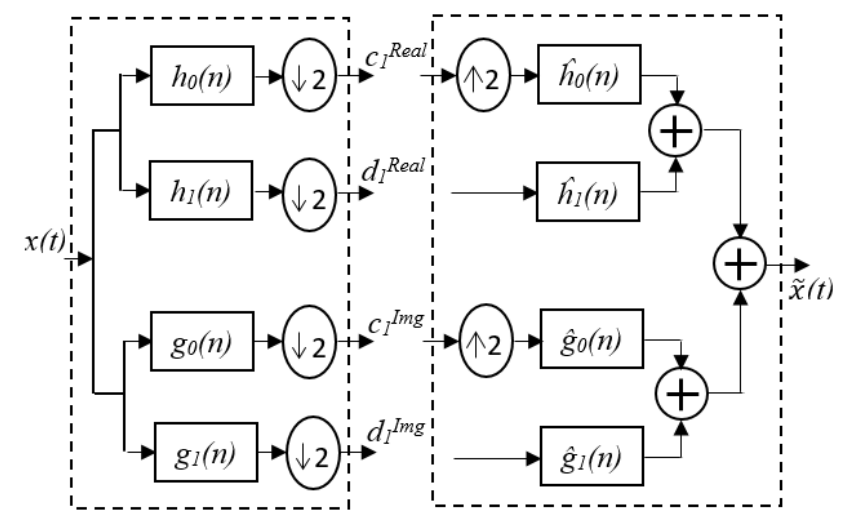

Figure. 3 Analysis and synthesis process of a onelevel dual tree complex wavelet transform

sample) filter bank are the two types of filter banks proposed by [15]. Odd/even filter bank exist some problems. Q-shift filter bank structure used in this implementation for denoising, and even filter lengths used after level one with no longer strictly linear phase [15].

Let noisy input signal $x(t)$ is derived after antenna array, $\psi_{h}(t)$ represents real / even and $\psi_{g}(t)$ represents imaginary / odd two wavelets with scaling functions are $\phi_{h}(t)$ and $\phi_{g}(t)$ respectively. The complex wavelet signal $\psi_{c}(t)$ becomes an analytic signal which forms Hilbert transform pair, and it is given by $[15,19]$ :

$$
\psi_{c}(t)=\psi_{h}(t)+i \psi_{g}(t)
$$

Now, real-part of wavelet coefficients and scaling coefficients of the tree be calculated by using the following formula [19]:

$$
\begin{aligned}
& w_{d}^{\mathrm{Re}}(n)=2^{\frac{d}{2}} \int_{-\infty}^{+\infty} x(t) \psi_{h}\left(2^{d} t-n\right) d t, \\
& S_{d}^{\mathrm{Re}}(n)=2^{\frac{D}{2}} \int_{-\infty}^{+\infty} x(t) \phi_{h}\left(2^{D} t-n\right) d t
\end{aligned}
$$

Where $d$ denotes the decomposition level $(d=1,2$, $3 \ldots D$ ), and $D$ represents the maximum value of the decomposition level. Similarly, for imaginary-part of wavelet coefficients and scaling coefficient of the tree calculated by using the following formula [19]:

$$
\left.\begin{array}{l}
w_{d}^{\operatorname{Im}}(n)=2^{\frac{d}{2}} \int_{-\infty}^{+\infty} x(t) \psi_{g}\left(2^{d} t-n\right) d t, \\
S_{d}^{\operatorname{Im}}(n)=2^{\frac{D}{2}} \int_{-\infty}^{+\infty} x(t) \phi_{g}\left(2^{D} t-n\right) d t
\end{array}\right\}
$$


With Eq. (6) and Eq. (7), the following are the complex wavelet coefficient and complex scaling coefficient of the tree denoted as:

$$
\left.\begin{array}{l}
w_{d}^{C}(n)=w_{d}^{\mathrm{Re}}(n)+i w_{d}^{\operatorname{Im}}(n) \\
S_{d}^{C}(n)=S_{d}^{\mathrm{Re}}(n)+i S_{d}^{\operatorname{Im}}(n)
\end{array}\right\}
$$

After the decomposition of the signal, the detail (wavelet) coefficients and scaling (approximation) coefficients are taken into ICA to remove the lower order coefficients and to improve the performance of denoising techniques.

Afterward, the denoised forward signal $D f n$ is extracted to get the complex wavelet coefficients and scaling coefficient by using inverse wavelet transform (reconstruction process) to obtain detail and approximation signal [19]. The detail signals are obtained using the reconstruction process,

$$
D S_{d}(t)=2^{\frac{d-1}{2}}\left\{\begin{array}{l}
{\left[\sum_{n=-\infty}^{+\infty} w_{d}^{\mathrm{Re}}(n) \psi_{h}\left(2^{d} t-n\right)\right]+} \\
{\left[\sum_{n=-\infty}^{+\infty} w_{d}^{\operatorname{Im}}(n) \psi_{g}\left(2^{d} t-n\right)\right]}
\end{array}\right\}
$$

Similarly, the approximation signals are obtained using the reconstruction process,

$$
A S_{D}(t)=2^{\frac{D-1}{2}}\left\{\begin{array}{l}
{\left[\sum_{n=-\infty}^{+\infty} S_{D}^{\mathrm{Re}}(n) \phi_{h}\left(2^{D} t-n\right)\right]+} \\
{\left[\sum_{n=-\infty}^{+\infty} S_{d}^{\mathrm{Im}}(n) \phi_{g}\left(2^{D} t-n\right)\right]}
\end{array}\right\}
$$

The reconstructed denoised signal $R e X$ calculated by summing the detail and approximation signals,

$$
\operatorname{Re} X=A S_{D}(t)+D S_{d}(t), d=1,2, \cdots, D
$$

\subsection{Independent component analysis algorithm}

Independent Component Analysis (ICA) is perhaps the most widely used method for performing blind source separation, and it is based on the assumption that the source signals are statistically independent $[16,17]$. This characteristic is used by ICA to estimate the signal of interest from the multipath signals.

As the standard wavelet transform, the DTCWT also generates correlated wavelet coefficients with their nearby region during decomposition of the signal at the wavelet transform [18]. If the wavelet coefficient has a large magnitude will possibly have

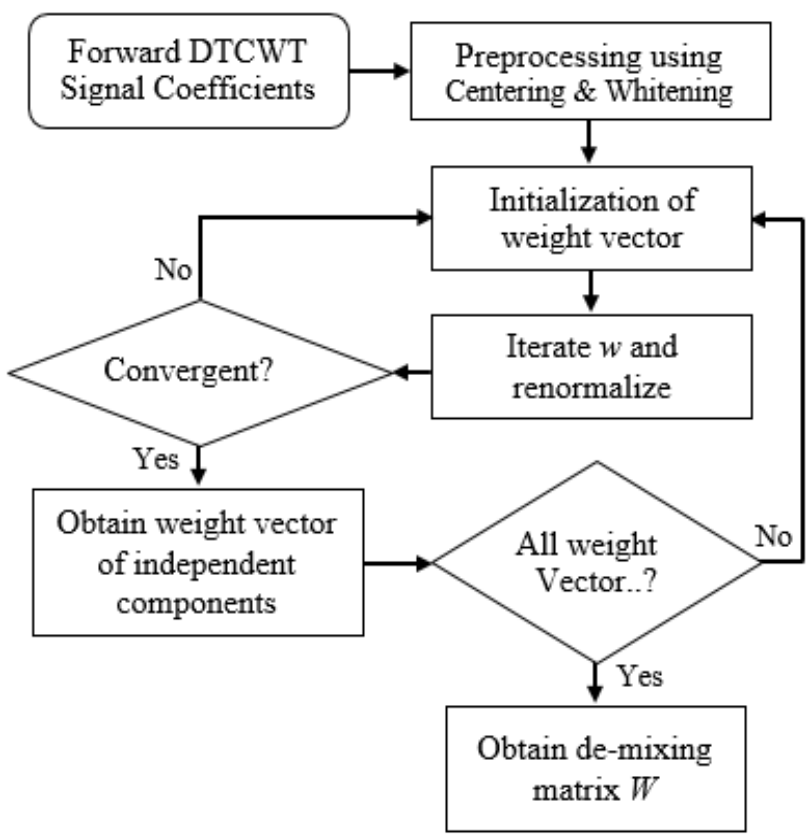

Figure. 4 Independent component analysis process

significant magnitude complex wavelet coefficients in the neighboring area. These neighboring coefficients acted as correlated wavelet coefficients and removed by using the ICA process [8, 16-17] described in Fig. 4.

The preprocessing using centering and whiting operation is carried out to increase the convergence rate. If $M$ sensors are used to receive the $N$ unknown mutually statically independent source signals, the mixed received signal vector is expressed as,

$$
X(t)=A \times S(t)
$$

To estimate the signal of interest, i.e., source signal from the mixed signal, the output of ICA expressed as,

$$
\begin{aligned}
& Y(t)=W \times X(t)=W \times(A \times S(t)) \\
& Y(t)=(W \times A) \times S(t)=I \times S(t)=S(t)
\end{aligned}
$$

Where ICA output $Y(t)$ is the estimation of unknown source signals $S(t), W$ is the de-mixing or weighted matrix, and $I$ is the Identity matrix. If any unknown source signal is multiplied with the identity matrix, the resultant is source signal from the observed mixed signal. It can be obtained based on the properties of the identity matrix in which diagonal elements are 1 in each row.

Choose initial weighted matrix $w=1$, and update $W$ with the derived iterative formula of the de-mixing matrix $W$ for $k$ independent components is as follows: 


$$
\begin{aligned}
& w_{k+1}=E\left\{X\left(w_{k}^{H} X\right) f\left(\left|w_{k}^{H} X\right|^{2}\right)\right\}- \\
& E\left\{f\left(\left|w_{k}^{H} X\right|^{2}\right)+\left|w_{k}^{H} X\right|^{2} f^{\prime}\left(\left|w_{k}^{H}\right|^{2}\right)\right\} w_{k}
\end{aligned}
$$

Renormalize by using $w_{k+1}=\frac{w_{k+1}}{\left\|w_{k+1}\right\|}$

Where $w$ is the raw value of the de-mixing matrix $W$, $f($.$) is the 1^{\text {st }}$ order derivative, and $f^{\prime}\left(\right.$.) is the $2^{\text {nd }}$ order derivative of nonlinear function $F($.$) . Iterate w$ using Eq. (16) for converges to obtain the weight vector of de-mixing matrix $W$ for all independent components $k=\left[\begin{array}{lll}w_{1} & w_{2} \ldots w_{n}\end{array}\right] x$.

\subsection{MUSIC algorithm}

The MUSIC algorithm proposed by Schmidt is a high-resolution Multiple Signal Classification techniques based on exploiting the eigen structure of the input array covariance matrix [2]. Space spanned by eigen vectors of array covariance matrix is divided into the principal eigen subspace (signal subspace) and the non-principal eigen subspace (noise subspace). The array steering vectors conforming to the source signals are orthogonal to the noise subspace. As the noise subspace is orthogonal to the signal subspace, these steering vectors are contained in the signal subspace. It should be noted that the noise subspace is spanned by the eigen vectors associated with the smaller eigenvalues of the correlation matrix, and the signal subspace is spanned by the eigen vectors associated with its larger eigen values [3]. From the elementary discussion in array signal model, this implies that $M-D$ of the eigen values of $R_{x}$ is equal to the noise variance $\sigma^{2}$. Then sort the eigenvalues of $R_{x}$ in decreasing order such that $\lambda_{0}$ is the largest eigen value and $\lambda_{M-1}$ is the smallest eigen value. Therefore,

$$
\lambda_{0} \geq \lambda_{1} \geq \cdots \lambda_{M-1}=\sigma_{n}^{2}
$$

The autocorrelation matrix $R_{x}$ is estimated from a finite data samples when all the eigen value corresponding to the noise power will not be identical. Instead, it will appear as a closely spaced cluster with the variance of spread decreasing as the number of samples used to obtain an estimate of $R_{x}$ is increased. Once the multiplicity factor $K$ of the smallest eigenvalue is determined, an estimated of the number of signals can be obtained as,

$$
\hat{D}=M-K
$$

The eigen vector associated with a particular eigen value $\lambda_{i}$ is the vector $q_{i}$ such that,

$$
\left(R_{x}-\lambda_{i} I\right) q_{i}=M-K
$$

For eigen vector associated with the $M-D$ smallest eigenvalue, we have

$$
\begin{aligned}
\left(R_{x}-\sigma_{n}^{2} I\right) q_{i} & =A R_{S} A^{H} q_{i}+\sigma_{n}^{2} I-\sigma_{n}^{2} I=0 \\
A R_{S} A^{H} q_{i} & =0
\end{aligned}
$$

Since $A$ has full rank matrix and $R_{S}$ is non-singular vector, this implies that

$$
A^{H} q_{i}=0
$$

Therefore, the eigen vectors associated with $M-D$ smallest eigen values are orthogonal to the $D$ steering vectors that make up $A$,

$$
\left\{a\left(\theta_{0}\right), \cdots, a\left(\theta_{D-1}\right)\right\} \perp\left\{q_{D}, \cdots, q_{M-1}\right\}=0
$$

We can estimate the steering vectors linked with the received signals by evaluating the steering vectors orthogonal to the eigen vectors associated with the eigenvalue of $R_{x}$ that is approximately equal to $\sigma_{\mathrm{n}}{ }^{2}$. The steering vectors corresponding to DOA lie in the signal subspace and are hence orthogonal to noise subspace. By searching through all possible array steering vectors to find those which are perpendicular to space spanned by the non-principal eigen vectors, the DOAs can be determined.

$$
v_{n}=\left[q_{D}, q_{D+1}, \cdots, q_{M-1}\right]
$$

As steering vectors consist of source signal which is orthogonal to the noise subspace eigen vectors, $a^{H}(\theta) V_{n} V_{n}{ }^{H} a(\theta)=0$ for $\theta$ corresponding to the DOA of a multipath component. Therefore, DOAs can be estimated by locating the peak of a MUSIC spatial spectrum given by,

$$
P_{\text {MUSIC }}(\theta)=\frac{1}{a^{H}(\theta) V_{n} V_{n}^{H} a(\theta)}
$$

Orthogonality between $a(\theta)$ and $V_{n}$ will minimize the denominator and hence will give rise to peaks in the MUSIC spectrum defined in Eq. (25). The number of largest peaks in the MUSIC spectrum correspond to the DOA of the signals impinging on the array. 


\subsection{Summary of DOA estimation using CW-ICA MUSIC algorithm}

1. The output of $M$ antenna element array is calculated using Eq. (1).

2. Forward wavelet transform of received antenna array signal is obtained using DTCWT. This decomposition process generates coefficients using Eq. (9).

3. ICA based thresholding is applied to these coefficients as described in Fig. 4. Weight vector $w$ is iteratively calculated using Eq. (16) to obtain de-mixing matrix $W$.

4. Denoised signal $\operatorname{ReX}$ is generated using inverse DTCWT of de-mixing matrix $W$ to obtain array covariance matrix $R_{x}$. This reconstruction process generates denoised signal using Eq. (12).

5. Eigen analysis of array covariance matrix to obtain DOA of uncorrelated, closely spaced, and coherent signal using Eq. (25) is carried out.

\section{Results and discussions}

In this section, simulation results on closely spaced coherent signals are presented to demonstrate the potential advantages of the proposed CW-ICA MUSIC algorithm compared to that of Waveletbased MUSIC [7], and classical MUSIC [2] algorithms respectively. The proposed technique is also compared with latest DOA estimation SSMUSIC [14] technique in terms of the probability of resolution with SNR and angular separation $(\delta \theta)$. The simulations have been carried out with Matlab R2014a. A uniform linear array of 12 antenna elements with the distance between antenna elements in the array is $0.5 \lambda$ is considered. Input signals are assumed to be of same power with 1000 snapshots.

\subsection{Spatial resolution analysis}

The performance of proposed DOA estimation technique is evaluated considering two closely spaced coherent signals with DOA of $\left[20^{\circ}, 22^{\circ}\right]$ are impinging on antenna array. The SNR is set to be $20 \mathrm{~dB}$ (very high). The evaluated spatial power spectrum is shown in Fig. 5.

From Fig. 5, we can conclude that the proposed technique CW-ICA music clearly resolves two closely spaced coherent signals. The proposed algorithm has accurate resolution and sharper peaks compared to wavelet MUSIC. The basic MUSIC algorithm fails to detect these two signal components. The wavelet-based MUSIC algorithm cannot distinguish their exact direction and has an

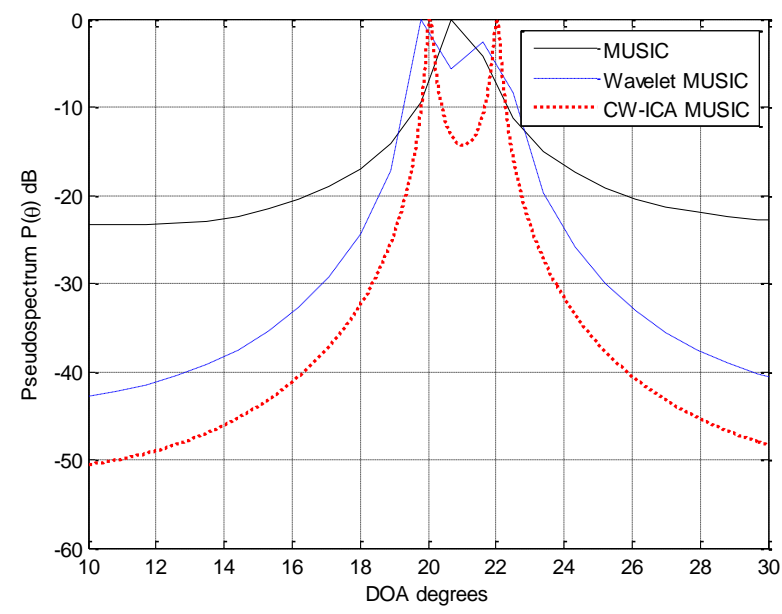

Figure. 5 Spatial spectrum for high SNR (20dB)

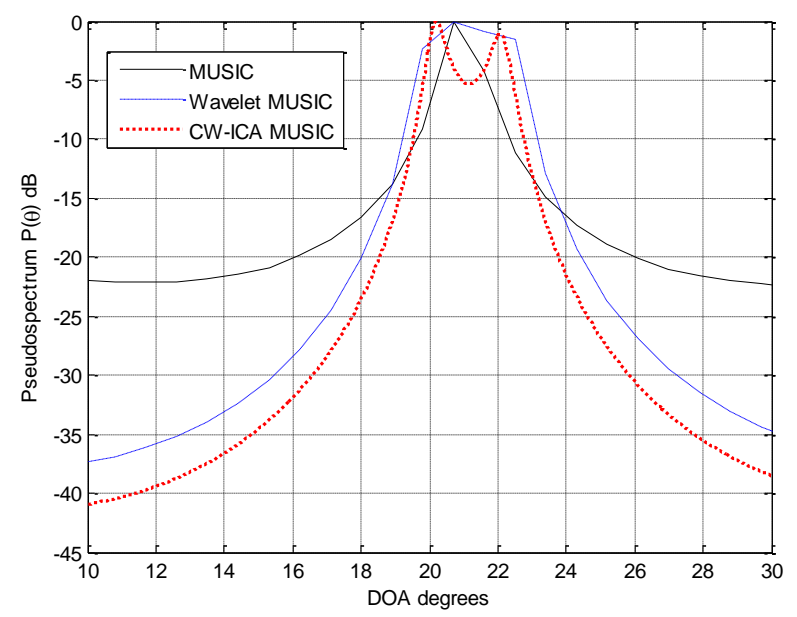

Figure. 6 Spatial spectrum for low SNR (OdB)

inferior resolution for two closely spaced coherent sources due to lack of shift-invariance property [15].

SNR is set to $0 \mathrm{~dB}$ (low), and rest of parameters unchanged. The results are depicted in Fig. 6. It shows that, for low SNR, the closely spaced coherent sources are appropriately resolved with relatively good resolution using the proposed approach. Basic MUSIC and wavelet MUSIC algorithms cannot distinguish the desired signals because of more noisy components in the received signal vectors.

SNR is further reduced to $-2 \mathrm{~dB}$ (very low), and the rest of parameters are unchanged. The results are depicted in Fig. 7.

Fig. 7 shows that for a very low SNR, the closely spaced coherent signals are resolved by the proposed approach. The proposed method performs remarkably even for low SNR and closely spaced signals since the preprocessing stage counteracts on largely correlated noisy coefficients. The preprocessing stage enhances the SNR of received signal and generates full rank of array covariance matrix. 


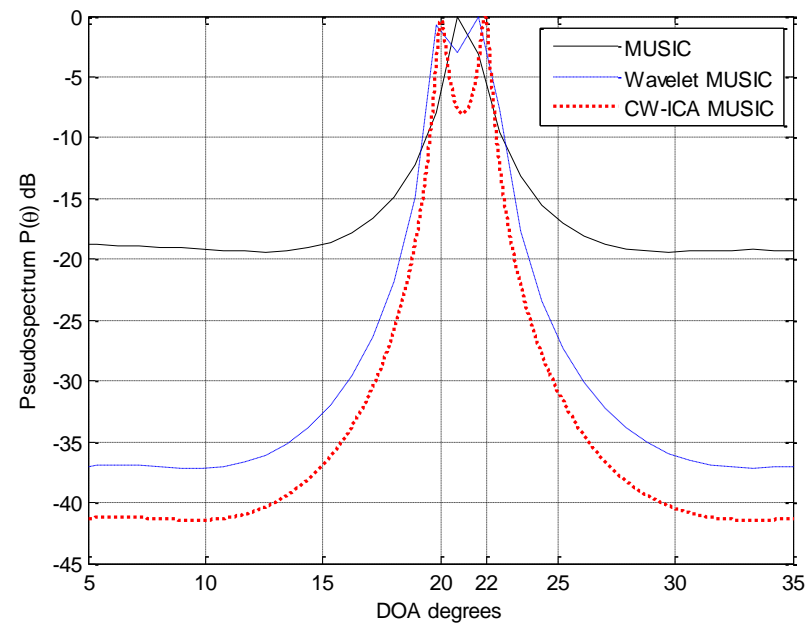

Figure. 7 Spatial spectrum for very low SNR (-2dB)

Performance of the proposed approach is reevaluated considering another two sets of closely spaced coherent signals with direction of arrival angles, $\left[20^{\circ}, 23^{\circ}\right]$ and $\left[30^{\circ}, 34^{\circ}\right]$. SNR is set to $0 \mathrm{~dB}$ (low), and rest of parameters unchanged. The DOA estimation results are depicted in Fig. 8. It is confirmed that the proposed CW-ICA MUSIC algorithm detects two sets of closely spaced coherent signals with relatively low SNR also.

\subsection{Effect of SNR on DOA estimation error}

The results depicted in Fig. 9 shows the Root Means Square Error (RMSE) plotted against SNR. SNR is varied from $-8 \mathrm{~dB}$ to $+8 \mathrm{~dB}$, and DOA estimated errors are obtained using 500 Monte Carlo simulation trails. Basic MUSIC algorithm is not considered in the error analysis since it does not deal with coherent signals. As the SNR goes on increasing, the error from the two algorithms gradually decreases to a lower value. At SNR $=0 \mathrm{~dB}$, proposed CW-ICA MUSIC algorithm has 0.1 RMSE compared 0.14 for wavelet based MUSIC.

\subsection{Effect of snapshots on DOA estimation error}

The results depicted in Fig. 10 show the RMSE plotted against a number of snapshots. SNR is set to $-2 \mathrm{~dB}$, and a number of snapshots are varied from 100 to 1000 . The DOA estimated errors are obtained using 500 Monto Carlo simulation trails. For the lower value of the snapshots, the proposed algorithm exhibits a low value of RMSE compared to that of wavelet-based MUSIC algorithm. The proposed CW-ICA MUSIC algorithm has 0.08 RMSE compared to 0.13 for wavelet based MUSIC when the number of snapshots is set to be 500. It indicates that the proposed method performs well even under poor enviornment conditions, for a low SNR, less number of snapshots and with moderate antenna elements.



Figure. 8 Spatial spectrum for two set of signals

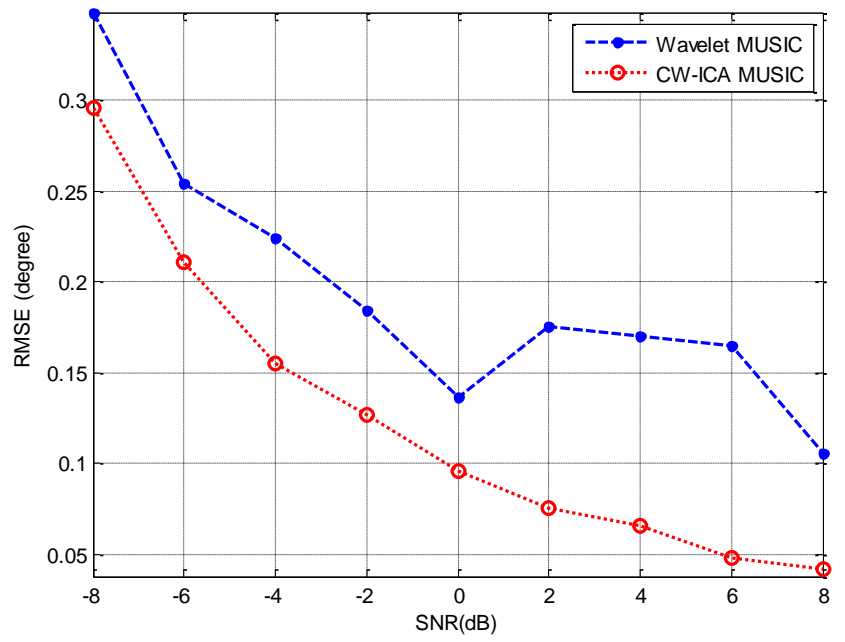

Figure. 9 RMSE vs. SNR

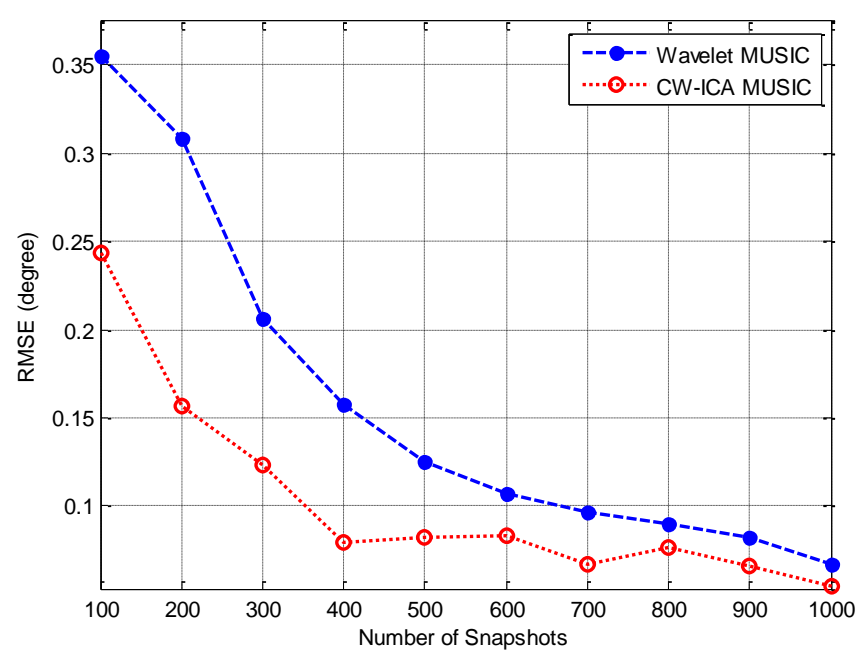

Figure. 10 RMSE vs. number of snapshots 


\subsection{Effect of SNR and angular separation on probability of resolution}

In this section, the DOA estimation performance of the proposed CW-ICA MUSIC method is compared with the latest method SS-MUSIC [14] to resolve closely spaced sources in terms of probability of resolution detection. The criterion for the probability of resolution is the ability to resolve closely spaced sources, and the two sources are said to be resolved in a given run expressed as [6],

$$
\left(\left|E \theta_{1}-T \theta_{1}\right|+\left|E \theta_{2}-T \theta_{2}\right|\right)<\left(\left|T \theta_{1}-T \theta_{2}\right| / 2\right)
$$

Where $T \theta_{1}, T \theta_{2}$ are the true DOAs and the $E \theta_{1}, E \theta_{2}$ are the estimated DOAs. The probability of resolution detection is calculated using the above criteria with the help of repeated 100 trials of Monte Carlo simulations with varying noise conditions. The results depicted in Fig. 11 shows the probability of resolution versus SNR for estimating two closely spaced uncorrelated sources with an agular separation of $2^{\circ}$. A number of snapshots is set to be 100 , and SNR is varied from $-30 \mathrm{~dB}$ to $+30 \mathrm{~dB}$. At low SNR conditions (0 to $5 \mathrm{~dB}$ ), the proposed method is observed to be performing better than SSMUSIC. At SNR of $5 \mathrm{~dB}$, the proposed method resolved the closely spaced signals with probability of resolution of 0.92 , and SS-MUSIC resolved with probability of resolution of 0.19 only. The proposed method works better than SS-MUSIC because of utilization of denoising stage with complex wavelet combined with ICA approach for thresholding the similar type of noisy coefficients in the neighboring region before DOA estimation.

To further evaluate the performance of the proposed method to resolve two closely spaced noncoherent sources, probability of resolution performance tested under different angular separation $(\delta \theta)$ as illustrated in Fig. 12. The SNR is set to be $7 \mathrm{~dB}$, and the angular separation between two closely spaced non-coherent sources is varied from 0.5 to 5 .

From Fig. 12, it is observed that, for a low angular separation, the performance of the proposed method is far better than SS-MUSIC. The angular separation from $2.5^{\circ}$, the proposed CW-ICA MUSIC method achieves probability of resolution of 1.00 indicates a $100 \%$ detection rate, while SSMUSIC obtains at $4.5^{\circ}$. Also, 0.9 probability of resolution is achieved for angular separation of $2^{\circ}$ compared to 0.3 of SS-MUSIC. Thus, the proposed method improves not only the resolution but also estimation accuracy compared to SS-MUSIC.

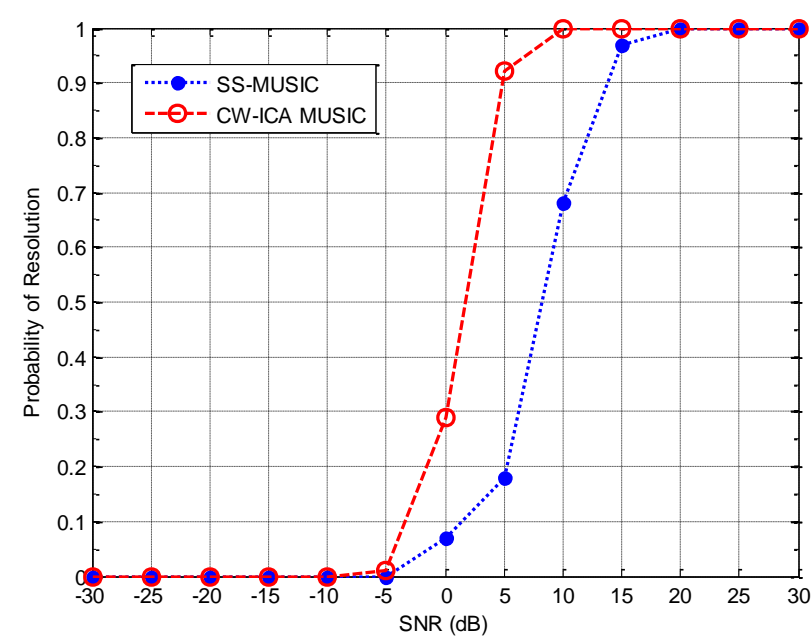

Figure. 11 Probability of resolution vs. SNR

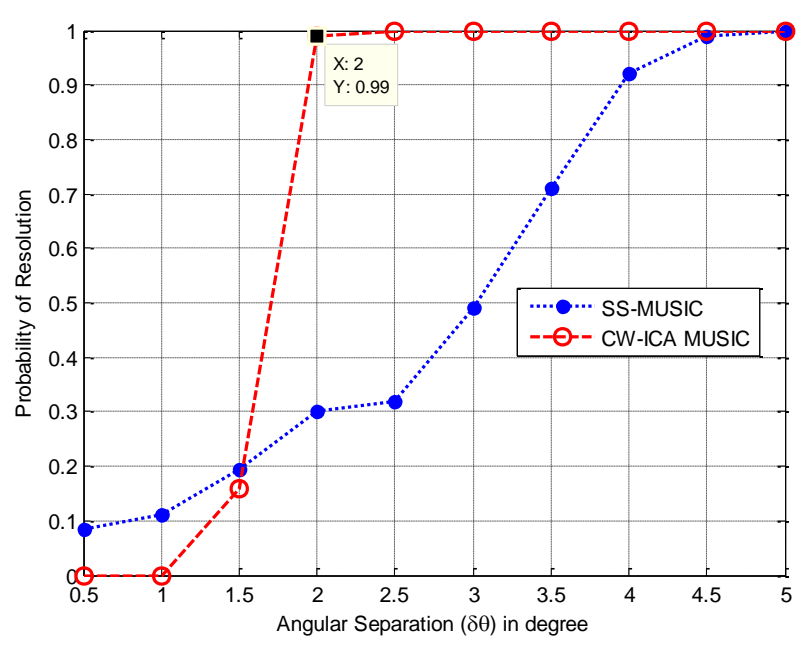

Figure. 12 Probability of resolution vs. angular separation

\section{Conclusions}

In this paper, DOA estimation for closely spaced coherent signal using Dual-tree complex wavelet transform and independent component analysis is investigated. A novel DOA estimation technique CW-ICA MUSIC is proposed. It includes a denoising stage before applying the signals to conventional DOA estimation algorithm. The simulation results exhibit the effectiveness of the proposed techniques compared to wavelet based MUSIC and SS-MUSIC. The proposed technique performs better even at low SNR, minimum number of antenna elements. This approach resolves the two closely spaced and coherent signals. A $2^{\circ}$ resolution is achieved with proposed algorithm for a low SNR of $-2 \mathrm{~dB}$. Also, signals with angular separation of $2^{\circ}$ are resolved successfully with 0.99 probability of resolution compared to probability of resolution of 0.3 with SS-MUSIC. Simulation results show that DTCWT-ICA based approach outperforms other 
techniques in multipath DOA estimation under low SNR, and closely spaced coherent signals scenarios. Investigations can be further extended to reduce RMSE for lower SNR by adopting population-based optimization algorithms.

\section{References}

[1] J. Liberti and T. Rappaport, Smart Antennas for Wireless Communications: IS-95 and Third Generation CDMA Applications, Prentice Hall, New Jersey, 1999.

[2] R. Schmidt, "Multiple Emitter Location and Signal Parameter Estimation", IEEE Transactions on Antennas and Propagation, Vol.34, No.3, pp.276-280, 1986.

[3] R. Roy and T. Kailath, "ESPRIT - Estimation of Signal Parameter via Rotational Invariance Techniques", In: Proc. of IEEE International Conf. on Acoustic, Speech Signal Process, Vol.37, No.7, pp.984-995, 1989.

[4] Y. Xue, J. Wang, and Z. Liu, "A novel improved MUSIC algorithm by wavelet denoising in spatially correlated noises", In: Proc. of International Symp. on Communication Information Technology, Vol.2, No.8, pp.499501, 2005.

[5] Z. Ye, Y. Zhang, and C. Liu, "Direction-OfArrival Estimation for Uncorrelated and Coherent Signals with Fewer Sensors", IET Microwaves, Antennas and Propagation, Vol.3, No.3, pp.473-482, 2009.

[6] L. Gan and X. Luo, "Direction-Of-Arrival Estimation for Uncorrelated and Coherent Signals in the Presence of Multipath Propagation", IET Microwaves, Antennas and Propagation, Vol.7, No.9, pp.746-753, 2013.

[7] X. Mao and H. Pan, "An Improved DOA Estimation Algorithm Based on Wavelet Operator", Journal of Communications, Vol.1, No.12, pp.839-844, 2013.

[8] L. Zhao, J. Xu, J. Ding, A. Liu, and L. Li, "Direction-Of-Arrival Estimation of Multipath Signals Using Independent Component Analysis and Compressive Sensing", PLoS ONE, Vol.12, No.7, pp.1-17, 2017.

[9] C. Chen, C. Jen, and A. Chang, "HighResolution DOA Estimation Based on Independent Noise Component for Correlated Signal Sources", Neural Computing and Applications, Vol.18, No.4, pp.381-385, 2009.

[10] G. Ma, Z. Sha, Z. Liu, and Z. Huang, "ICA Based Direction of Arrival Estimation of Uncorrelated and Coherent Signals with
Uniform Linear Array", Signal, Image and Video Processing, Vol.8, pp.543-548, 2014.

[11] A. Chang and C. Jen, "Complex-Valued ICA Utilizing Signal-Subspace Demixing for Robust DOA Estimation and Blind Signal Separation", Wireless Personal Communication, Vol.43, pp.1435-1450, 2007.

[12] M. Golroudbari and M. Allam, "Direction of Arrival Estimation in Multipath Environments Using ICA and Wavelet Array Denoising", In: Proc. of International Conf. on Application of Information and Communication Technologies, Baku, pp.1-5, 2011.

[13] X. Luo, P. Wei, L. Gan, and H. Liao, "Improved Direction-Of-Arrival Estimation for Uncorrelated and Coherent Signals in the Presence of Multipath Propagation", IEICE Transactions Fundamentals, Vol.E98-A, No.3, pp.881-884, 2015.

[14] S. M. Hadj Irid, S. Kameche, and S. Assous, "A Novel Algorithm to Estimate Closely Spaced Source DOA", International Journal of Electrical and Computer Engineering, Vol.7, No.4, pp. 2109-2115, 2017.

[15] I. Selesnick, R. Baraniuk, and N. Kingsbury, "The Dual-Tree Complex Wavelet Transform", IEEE Signal Processing Magazine, Vol.22, No.6, pp.123-151, 2005.

[16] E. Bingham and A. Hyvärinen, "A Fast FixedPoint Algorithm for Independent Component Analyses of Complex Valued Signals", International Journal of Neural Systems, Vol.10, No.1, pp.1-8, 2000.

[17] A. Hyvärinen and E. Oja, "Independent Component Analysis: Algorithms and Applications", Neural Networks, Vol.13, No.4, pp.411-430, 2000.

[18] G. Chen and W. Zhu, "Signal Denoising Using Neighbouring Dual-Tree Complex Wavelet Coefficients", IET Signal Processing, Vol.6, No.2, pp.143-147, 2012.

[19] X. Yu, W. Liang, L. Zhang, H. Jin, and J. Qiu, "Dual-Tree Complex Wavelet Transform and SVD Based Acoustic Noise Reduction and Its Application in Leak Detection for the Natural Gas Pipeline", Mechanical Systems and Signal Processing, Vol.72-73, pp. 266-285, 2016. 